\title{
Multilinguales
}

$12 \mid 2020$

Varia 2019

\section{Autobiographie et écriture de l'intime dans les romans de Malika Mokeddem}

Autobiography and writing of the intimate in the romans of Malika Mokeddem

Fizia Hayette Boulahbel Mokhtari.

\section{(2) OpenEdition}

Journals

Édition électronique

URL : https://journals.openedition.org/multilinguales/4157

DOI : $10.4000 /$ multilinguales. 4157

ISSN : 2335-1853

Éditeur

Université Abderrahmane Mira - Bejaia

Référence électronique

Fizia Hayette Boulahbel Mokhtari., « Autobiographie et écriture de l'intime dans les romans de Malika Mokeddem », Multilinguales [En ligne], 12 | 2020, mis en ligne le 14 février 2020, consulté le 01 juillet 2021. URL : http://journals.openedition.org/multilinguales/4157 ; DOI : https://doi.org/10.4000/ multilinguales. 4157

Ce document a été généré automatiquement le 1 juillet 2021.

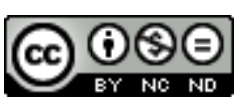

Multilinguales est mise à disposition selon les termes de la Licence Creative Commons Attribution Pas d'Utilisation Commerciale - Pas de Modification 4.0 International 


\title{
Autobiographie et écriture de l'intime dans les romans de Malika Mokeddem
}

\author{
Autobiography and writing of the intimate in the romans of Malika Mokeddem
}

Fizia Hayette Boulahbel Mokhtari.

1 Des études universitaires ont été menées notamment par Anna Robeson Burr ${ }^{1}$ qui a effectué des observations sur un échantillon d'une centaine d'autobiographies et a tenté d'établir des critères assez édifiants sur l'écriture de l'intime. Les chercheurs ont donc établi un classement des écrits intimes selon des critères pas toujours évidents à appliquer sur des écrits autobiographiques: ce classement selon "la religion, la nationalité, le sexe, la situation sociale ou économique des différents auteurs» (Hubier, 2003 : 68) a révélé plusieurs exceptions, c'est dire que tous les autobiographes n'écrivent pas leur vie intime de la même façon. Les chercheurs posent la problématique de l'écriture de l'intime; tel Philippe Lejeune dès les années 70, qui évoque l'idée de la cure analytique, soit celle de "dissocier l'image que le sujet se fait de lui-même pour lui faire reprendre conscience des événements qui sont à l'origine de sa névrose "(Hubier, $2003:$ 71), alors l'écrit autobiographique est une "élaboration, synthétique du sens qui récupère et structure les souvenirs dans un récit très consciemment dirigé, comme si l'intimiste s'arrêtait précisément là où commence le travail d'analyse »(Hubier, 2003 : 71).Philippe Lejeune pose la question sur un auteur qui, écrit ses souvenirs et se dit «Ecrire ses souvenirs, est-ce simplement décrire ce qu'on voit dans sa mémoire? (37), il pense que tout pousse le romancier à réinventer le passé ; il l'explique par le fait que « la mémoire offre un canevas sur lequel broder, des bribes de mélodies à harmoniser, des ruines à restaurer. » (37). Et c'est là encore une difficulté qui s'ajoute à l'écheveau que nous voulons démêler si nous pouvons nous exprimer ainsi. Philippe Lejeune tente d'expliquer en disant que le romancier joue avec son passé, et que c'est indispensable pour « communiquer l'émotion au lecteur. Dans ce travail de réinvention, les modèles appris ont un grand rôle. Tout moyen est bon, s'il contribue à produire l'émotion" (38). Ainsi, nous allons voir dans quelle mesure ces études faites sur l'écriture autobiographique, pourraient nous aider, dans une 
certaine mesure, à répondre à la question comment se manifeste l'écriture intime dans les romans de Malika Mokeddem?

Malika Mokeddem commence son roman autobiographique La Transe des insoumis par cette épigraphe: "Pour mon père ce livre qu'il ne lira pas.", son père est le premier homme de sa vie avec qui elle aura le plus de maux et le moins de mots à lui dire, mais de qui elle a tiré ce qu'elle a considéré comme son tribut de la liberté. Et c'est pour la liberté qu'elle a lutté toute sa vie. Mais Malika Mokeddem ne va lutter seulement contre les hommes de sa famille, elle va aussi livrer un combat sans fin avec celle qui l'a mise au monde.

3 Pour tenter de répondre à la problématique de l'écriture de l'intime que l'on retrouve dans certaines autobiographies, l'examen des écrits de Malika Mokeddem portera d'abord sur la difficulté de raconter sa vie puis nous verrons que dans ses textes, elle nous livre certains détails sur sa famille, des secrets, notamment sa relation conflictuelle avec ses parents qu'elle condamne, en faisant de longs réquisitoires et dénonce les injustices perpétuées par ses proches. Elle révèle sans tabous les relations qu'elle avait entretenues avec les hommes : son mari, ses amis et ses amants. Et enfin, le lecteur est pris comme témoin dans son discours; où il découvre son vécu rédimé par la fiction, la pousse inexorablement vers un exil qu'elle assume et qui la renvoie souvent vers sa condition d'apatride.

\section{Dire l'indicible dans l'écriture de Mokeddem}

May Georges ${ }^{2}$ a effectué des études sur des textes autobiographiques et a pensé que l'écriture de soi est bien plus complexe d'autant plus que la classification selon les origines, la famille, l'éducation, la profession de l'écrivain ne sont pas forcément les seules explications à donner, même le critère de l'âge avancé de l'écrivain et sa notoriété ne semblent pas être des critères fiables. Selon G May, les écrits autobiographiques peuvent être écrits par n'importe qui et à n'importe quel âge (pas forcément avancé, comme on pourrait le croire) selon lui « la vocation autobiographique tien[drait] probablement plus aux conditions culturelles et historiques qu'aux particularités individuelles "(69) Nous pouvons dire qu'il y a en effet, dans l'écriture autobiographique de Malika Mokeddem ${ }^{3}$ une part de ce qui a été énoncé dans ces études littéraires, et en même temps, cela se révèle difficile à affirmer complètement parce que chez Assia Djebar par exemple, nous pourrions dire que ce qui justifie son autobiographie sont les deux aspects cités plus haut, or nous avons expliqué que malgré le cadre social compliqué et austère à la femme, d'une manière générale, il y a dans cette forme d'écriture, une forme de lutte, où les aspects intimes de sa vie ne sont pas toujours narrés de manière directe et nous avons même relevé que la vie intime est alambiquée à raconter ; revenir à la ponctuation, les points de suspension très nombreux dans les romans d'Assia Djebar montrent bien que l'intime est difficile à raconter mais est-ce que cela a un rapport avec le cadre social oui, probablement, avec l'éducation sans doute- avec les particularités individuelles, oui, il y a de fortes chances.

Cependant, nous avons un contre exemple, dans le cas de Malika Mokeddem; elle a exposé elle-même les motivations de son écriture autobiographique où elle fait "l'apologie de soi, se défend des accusations injustes et témoigne " (Hubier, 2003: 69) des relations qu'elle avait eues avec les autres. 


\section{Les maux de la mère}

6 Christiane Achour dit au sujet de l'image de la mère dans la littérature de Malika Mokeddem qu'elle est « de l'ordre du rejet : la mère est le contre-modèle, celle à qui il ne faut ressembler en rien, le modèle répulsif alors que le père, malgré tout est préservé »(Achour-Chaulet, 2007: 45) Malika Mokeddem sera en perpétuel confrontation avec sa mère, dès son jeune âge et surtout accentuée après son entrée à l'école, une fracture s'installe entre les deux femmes: Malikadira dans une interview, une citation tirée d'un entretien qu'elle avait donné à Montpellier :

Je pense que la mère ... elle est absente. Elle n'existe pas [...] j'ai mis du temps à m'en rendre compte. La mère n'est jamais là. Même dans $\mathrm{N}^{\prime} \mathrm{zid}^{4}$, la mère a mis au monde une fille et elle est repartie en Algérie, carrément vers une autre terre. Je pense que c'est quelque chose d'inassouvi [...] donc je ne peux que l'inventer (Bénayoun-Szmidt et al, 2007 : 280)

7 Corinne Blanchaud dans son article sur l'écriture de Malika Mokeddem dit que «la puissance des mots est l'un des motifs de son espace autobiographique puisqu'ils lui ont permis d'échapper au destin déterminé par son milieu d'origine. » (317). La romancière dresse un sombre portrait de sa mère, elle met en place une image d'une mère très éloignée du modèle maternel affectueux comme pour rappeler que le malheur et les combats menés viennent de ce rejet « originel ». Ce qui va séparer les deux personnes après la grand-mère, c'est l'école. La petite fille, qu'elle soit Malika, Leila, Selma ou Nora, dès son entrée à l'école, elle va considérer la nouvelle langue qu'elle apprend comme une arme. Et l'amour qu'elle vouera aux mots appris et recopiés soigneusement sur le cahier, vont l'éloigner définitivement de sa mère :

Je m'applique à dessiner les pleins et déliés, prononce de temps en temps les lettres à voix haute, continue de ressasser leur sonorité dans ma tête pour ne pas m'attirer trop de railleries, rêve sur les empreintes de mon buvard. Longtemps. Ma mère me jette parfois un regard impatient. Elle a tant besoin d'être secondée. Mon échappée est trop longue. Moi, je m'extasie à admirer le livre ouvert, le cahier sur lequel je recopie. [...] (Malika Mokeddem, 2003 :47.48)

Malika Mokeddem affiche ouvertement cette "désobéissance " (Achour-Chaulet, 2007 : 151) sans doute pour attirer l'attention de la mère, mais pas si sûre. Elle veut plutôt plaire à son père, à celui qu'elle a aimé finalement, puisque le retour à la fin de $L a$ Transe des Insoumis se fait vers lui essentiellement. Nous pouvons constater comment dans ses romans, elle a toujours cherché des pères de substitution, et cette hypothèse vient du fait qu'elle a tissé « un réseau masculin » qui montre « directement cette constante par le regard de l'homme "(Achour-Chaulet, $2007: 151.152)$. L'univers de l'école et des livres seront le sanctuaire où la mère est pratiquement exclue. La narratrice ne veut pas ressembler à sa mère, du fait qu'elle ait mis au monde beaucoup d'enfants, elle ne veut pas enfanter et la correspondance à l'enfantement est évoqué dans plusieurs de ses romans, Je dois tout à ton oubli, Mes hommes, N'zid, elle se moque même des femmes qui font croire à la société que les filles qui se font engrosser sont heureuses; pour elle, elles sont hypocrites et parler de « bien-être fœtal » est une invention. On voit même une certaine objectivité scientifique expliquer le phénomène de l'enfantement, et elle associe son refus de donner naissance à des enfants par la déconvenue de sa naissance :

Les postulats des revues de médecine reviennent percuter dans ma tête les clichés comparant le sommeil dans un bateau au bien-être du fœtus dans son liquide amniotique. Je n'ai jamais cru à cette notion de bien-être fœtal. Elle me semble même des plus suspectes avec des relents de moralité. Et comment rapprocher 
l'extrême liberté éprouvée en bateau à l'état de dépendance totale du fœtus ? La

fusion idéale de l'embryon à la mère n'est qu'organique. (Mokeddem, 2003 : 95)

Elle associe son refus d'enfanter par le fait même qu'elle avait subi un rejet de la part de sa propre mère. Elle raconte avec une grande amertume comment les femmes prient Dieu pour avoir un garçon, parce qu'avoir une fille est perçu par les femmes comme une malédiction, et dans son discours, le lecteur est mis dans la confidence intime de la narratrice :

Là-bas, j'avais si souvent entendu des femmes enceintes gémir et implorer Allah afin que les enfants qu'elles portaient fussent de sexe masculin. En se malaxant le ventre, les yeux vrillés par leur conjuration. Je me dis maintenant qu'il y avait de quoi communiquer cette angoisse aux foetus, à tous, quel que fût leur sexe, si l'on suppose qu'ils n'en ont pas encore conscience à ce stade-là. [...] Du reste qu'importe ce souvenir inaugural, si souvenir il y a. De toute façon les voix de celles qui ont assisté : mère, grand-mère et tantes se chargeront plus tard de ressasser à ces filles leur traumatisme à elles pour mieux leur enfoncer dans le crâne leur sentiment d'infériorité. (Mokeddem, 2003 : 95.96)

Dans ce paragraphe, c'est avec un certain cynisme que la romancière décrit la situation de sa naissance et celle des autres enfants. Dans ces petits épanchements, elle met à témoin le lecteur de la situation des femmes qui ont peur d'avoir des filles, de peur d'être répudiées. Elle reprend cette relation mère-fille dans N'zid :

"Où a-t-elle entendu l'absurde analogie entre ce sentiment et celui de l'embryon dans son liquide amniotique ?" " Niaiserie. Il n'y a pas plus suspect que cette idée de bien-être foetal. Cette pensée lui noue la gorge. [...] ${ }^{\text {"n }}$ (Mokeddem, $2001: 28.29$ ).

11 L'écriture autobiographique, chez Malika Mokeddem indique la volonté de retracer "un parcours intellectuel exemplaire sur la minutieuse narration d'une existence humaine " (Miraux, 1996: 43) l'esprit scientifique est ainsi le fond de sa nature, elle le fait plusieurs fois dans ses écrits fictifs aussi.

Le rejet est marqué par la colère. Et le regard sévère, porté sur la relation ombilicale entre la mère et son enfant, montre la relation conflictuelle qu'elle a eue ou qu'elle dit avoir supporté toute sa vie. Christine Detrez dit que de "façon plus générale et quotidienne, la femme est ramenée, résumée à son corps, et plus spécifiquement à la valeur procréatrice» (70), ce que veut réfuter Malika Mokeddem dans son discours. Elle veut faire en sorte que sa vie ne corresponde pas au modèle de vie à l'algérienne qui consiste à se marier, et à accoucher tout de suite après. Nous pensons que même son anorexie est une tentative de résistance pour ne pas subir le châtiment de la société, son anorexie et son insomnie sont son combat. Elle ne veut pas qu'on la voit comme une femme en âge de se marier mais rester jeune fille et elle parvient lors de l'épisode du mariage arrangé, à fuir et à retarder l'échéance.

La vision qu'elle porte sur sa mère est une attention chargée de reproches et la réciprocité dans la critique dénote qu'elle sait mieux ce qui se passe et qu'elle possède le pouvoir de l'expression parce qu'elle se sent la cible d'une dictature maternelle. Et la parole et le pouvoir autoritaire ne font pas bon ménage car " le terroriste est misologue $e^{6}$ " (Miraux,2005: 41) La mère ne parle pas, elle est dans la gestuelle fracassante, elle montre sa colère en faisant du bruit, et son regard suffit à faire réagir la narratrice :

Le petit déjeuner prêt, ma mère vient sonner le clairon. En quelques minutes, tout le monde est debout. Sauf moi. [...] Mais ma mère ne m'accorde guère de répit. Son café à peine avalé, elle s'attaque aux premières tâches du manège : plier toutes les strates composant les couchages, les ranger en de laborieux édifices verticaux contre les murs, laver les plaids souillés d'urine, rincer les nattes en alfa. [...] Même 
le piédestal du lit, plus tard, ne m'a pas épargné ce tintamarre du matin. [...] Je me résigne à me lever, les paupières lourdes de manque de sommeil et l'œil noir de ressentiment. (Mokeddem, $2003: 115.116)$

13 Nous sommes face au cas de la "résolution des contraires» (Miraux, $1996: 41$ ), c'est un épisode de la vie de toute la famille qui semble insupportable pour la narratrice, et cette situation va se poursuivre avec la mère, en affrontement, le mot «guerre » est lancé dans son discours, le lecteur est témoin des instants d'une rare violence entre une mère et sa fille :

Mon appropriation de cette chambre donne le coup d'envoi de la guerre, jusqu'à ce jour larvée, entre ma mère et moi. Chaque matin, elle abandonne épisodiquement ses besognes pour venir tambouriner avec hargne contre la porte: "Hé! L'Américaine! Il y a du travail qui t'attend. Lève-toi !» [...]: chaparder encore quelque somnolence loin du tapage et des querelles. [...] L'aversion complète du sommeil inaugure la métamorphose $d u$ refus en résistance. Elle scelle ma détermination à ne pas me laisser transformer en esclave de mes frères. (Mokeddem, 2003 : 116)

14 Tournée vers une vision nouvelle de la société, elle se délecte de sa singularité pour proposer des vues nouvelles de la condition de la femme algérienne; de sa position de fille aînée qui a des devoirs et des obligations et non des droits tous comme ces frères qui, eux sont élevés et traités différemment. Elle a des ambitions et des aspirations qu'elle estime légitimes et elle a conscience que la femme algérienne est l'ennemie de la femme algérienne, que sa condition est tributaire des agissements qu'elle adopte. Elle prend son cas particulier pour lui donner un caractère collectif et général :

Eux, ils passent leur journée à jouer, à nager à la piscine. Le soir, ils peuvent aller au cinéma. De toute façon, ils sont non seulement libres mais encore choyés, adulés. Moi, je n'ai droit à rien de cela. Je devrais seulement servir, obtempérer et me taire. Taire même le chagrin que m'infligent tant de ségrégations dans l'affection. [...] Je me ferais tuer plutôt que de prêter un petit doigt à quelque rangement. C'est, je crois, à cette époque que j'ai commencé à prendre conscience du regard glacé que ma mère jette sur moi, de la constance de ses aboiements, de l'absence de mots affectueux ou rassurants. (Mokeddem, 2003 :116)

15 Le conflit entre la mère et la fille est repris avec la même acuité dans le roman Je doi stout à ton oubli, Malika Mokeddem dira sur ce roman il « est le texte le plus douloureux à écrire sur ma mère » (Bouriche, 2011 : 20). Le combat avec la mère se mesure par la force des cris et des regards échangés, la narratrice ne cache pas au lecteur ce qu'elle a pensé de la situation, la narratrice se poste dans la position de celle qu'on accable et où elle "se défend, au nom des valeurs du juste et de l'injuste.» (Hubier, 2003: 68) elle fait le reproche à la mère d'être une mère au jugement partial qui la révolte au plus haut point :

Je ne restituerai pas de sitôt la clef de la fameuse pièce. Je hurle ou détale quand ma mère tente de me l'arracher. Mes cris l'inhibent, la tétanisent d'effroi. Sauf à rugir comme une bête blessée sous les coups de la mort, une fille ne crie jamais, surtout pas de rébellion. Moi, j'ai appris ça, la force du cri. Je sais son poids de honte et d'interdit. [...] J'en ai mesuré l'impact dans les yeux de ma mère. J'en ai apprécié la puissance répulsive. (Mokeddem : $2003: 117$ )

16 La narratrice se rappelle, enfant, comment « la couche collective $»^{7}$ l'oppressait au point de se réveiller chaque soir et de se retrouver avec plaisir les bras protecteurs de sa grand-mère et non ceux de sa mère. La narratrice- personnage-auteur en relatant par écrit cette auto-analyse découvre le sens de ses souffrances et finit même par être heureuse de trouver la joie ailleurs que dans les bras de sa mère : 
L'idée saugrenue, je le sais, que l'abandon du sommeil me la rendait peut être accessible ; la certitude d'être rabrouée m'arrête [...] l'interpellation de ma grandmère me donne des ailes. Je vole vers elle (Mokeddem, 2003 : 30)

jamais eu le geste avenant que la petite fille aurait souhaité voir venir. La mère au contraire n'aime pas l'influence de la grand-mère sur elle, petite fille rebelle déjà... elle exige d'elle de rester dormir avec ses frères et sœurs. Noctambule, en assistant à cette scène étrange pour elle, petite, mais adulte, elle sait qu'elle a assisté à une scène intime qu'elle n'aurait pas pu voir si la maison n'était pas petite et trop étroite, grande, la maison ne permettait pas cette promiscuité, et mettre la petite fille dans une position aussi sensible ; en tout cas, cet épisode lui permettra d'acquérir sa liberté et l'insomnie n'était plus interdite puisque dorénavant elle allait partager sa couche avec sa grandmère.

La mère et elle ont une relation très tendue et compliquée. Cette femme est décrite par la narratrice comme une femme toujours enceinte et qui n'a pas été vraiment maternelle avec sa fille. La narratrice déclare sa mère illettrée et toujours "pleine ». Dans son passé, les souvenirs liés au souvenir de "sa haine pour la mère » est associée fatalement à la soif d'amour et c'est l'écriture qui viendra panser les blessures de cette solitude ombilicale. On peut dire que l'écriture a un rôle cathartique évident : elle dira dans un autre roman intitulé Mes Hommes :

Il y a tant de solitude dans la vie ; sans famille en dépit de parents encore vivants, d'une nombreuse fratrie. Sans enfants par choix. Seule entre deux pays [...] Seule entre l'écriture et la médecine [...] (Mokeddem, 2005 :214)

Dans les romans « autobiographiques »: Mes hommes, N'zid, La transe des insoumis, Je dois tout à ton oubli, et même ceux qui ne sont pas autobiographiques en tout cas affichés comme tels, Les hommes qui marchent, La Désirante, Des rêves et des Assassins, Le siècle des sauterelles, Malika Mokeddem espère peut-être en relatant par écrit les souvenirs, découvrir le sens de ses souffrances: la relation avec sa mère déterminera son devenir, et sarelation avec le reste de la famille. Elle est exilée géographiquement et émotionnellement :

Lucide, Selma observe chacun de ses frères et sœurs qui avancent vers elle [...] l'expression de Goumi, à propos de sa relation à la fratrie, lui revient en tête : Vous n'avez pas eu la même mère ". Morte, la mère n'en demeure pas moins là, entre eux. Pas la même. Elle a façonné le regard que les autres portent sur Selma. Et le rapport de l'ainée à cette contrée. Elle a transformé son enfance en une longue fugue. Elle a forgé son refus de l'enfantement. Elle n'a jamais eu de mère et elle ne sera jamais mère (Mokeddem, 2008 :38)

Selma personnage différent de la narratrice- auteur, cependant, on identifie clairement les problèmes relationnels qu'elle a avec sa mère; Malika Mokeddem emprunte par la voie du roman pour explorer un pan douloureux de sa mémoire. L'image de la mère est toujours celle qui a laissé sa fille même dans le roman N'zid où la mère semble être une mère victime de folie et séparée de son mari et de sa fille, Mokeddem avec cynisme reprend l'abandon qu'elle avait connu avec sa véritable mère :

La seule chose importante, c'est qu'elle nous ait appelés papa et moi. Par-delà les décennies de mutisme. Par-delà la démence...Au seuil de la mort...Tu parles, Zana! Je n'ai rien entendu...Hagitec-magitec ${ }^{8}$ ! Mon père tailleur de pierres et de courants m'a donné la mer entre deux langues. De l'autre rive, la mère, elle, a mis ma vie entre elle et moi (Mokeddem, $2001: 147$ ) 
arratrice est pensive et mélancolique où l'on décèle une certaine colère, nous voyons cela à l'expression «tu parles", une expression qui montre qu'elle ne croit pas un mot de Zana, une vieille femme qui aurait pu être sa grand-mère, elle lui ressemble à plus d'un point ${ }^{9}$, cette grand-mère qui raconte des histoires avec cette expression que Malika Mokeddem reprend dans Mes Hommes et La transe des insoumis et Les Hommes qui marchent : «Hagitec-magitec. ». L'image de la mère est celle qui a abandonné son enfant, comme elle semble avoir été abandonnée dans La Transe des Insoumis, Mes Hommes et Les hommes qui marchent.

Durant des années, elle n'a reçu que sa mère et même sa venue de France n'est justifiée que pour l'argent qu'elle doit lui donner pour ses frères et sœurs. Aucune question n'est posée par sa mère, elle ne lui demande jamais de ses nouvelles comme le ferait une mère attentive à son enfant, c'est encore des entretiens houleux qui ravivent la colère et l'incompréhension chez la narratrice :

Soudain, dans le brouhaha qui m'étourdit, me parviennent les lamentations de ma mère : " Tu continues à tout faire pour rendre tes cheveux d'alfa ! " Elle a ce regard en biais qu'elle a toujours posé sur moi. Laisser mes cheveux tirebouchonner au gré de leur fantaisie l'a toujours chagrinée. J'acquiesce, la moue goguenarde.[...] Décidément, j'aurai toujours quelque chose de travers [...] premières paroles, premiers constats. Je ne suis pas conforme à ce qu'elle se juge encore en droit d'attendre de moi. Même à mon âge! [...]" Mes pigments ne viennent pas de ton côté. J'aila peau tannée, le cheveu tordu par une goutte de sang noir descendant des aïeux de grand-mère " (Mokeddem, 2003 : 240.241)

L'auteure montre clairement que son mal de vivre est antérieur à son départ du pays. Son manque de communication avec sa mère est lié au fait qu'elle était plus proche de sa grand-mère que de sa mère ; le silence de sa mère s'est installé quand très jeune, elle avait mis entre elle et sa mère ses livres : « quand elle m'observe, j'ai l'impression qu'elle voit une martienne tant dans ses yeux la panique verrouille l'incompréhension" (Ibid.182).La narratrice s'auto-analyse, elle explique son drame: pour elle:»c'est l'oubli originel » (Ibid.182) elle ajoute dans le dosage du drame, «il est ma résilience. Il est à l'origine de tout. De ma relation avec ma mère » (182) son bienêtre, elle va le chercher en elle, dans son écriture et ce n'est que dans N'Zid qu'elle pense trouver son « intégrité » (182) :

J'avais un moment espéré qu'elle allait enfin s'inquiéter de ma vie à moi. Me demander seulement si j'étais bien. Comment je vivais ma solitude. Ils savent évidemment que nous nous sommes séparés Jean-Louis et moi voilà plus de sept ans. [...] Moi, je ne sais pas d'où elle me regarde, d'où elle me parle.[...] " je vous ai apporté de l'argent bien sûr. Je vais te le donner. Mais je n'ai pas cette somme. Tiens. Ça aussi c'est pour toi "(Mokeddem, $2003: 242.243$ )

En devenant célèbre, la narratrice est sollicitée par sa mère et ses nombreux frères et sœurs mais elle ne veut pas les aider; sa famille ignore tout d'elle et de sa situation sociale et financière. Dans son exil, elle ne cesse de penser aux siens et à compter les années de silence, et rien ne lui indique qu'un changement est pour bientôt :

Jamais ils ne comprendront que je ne veuille pas travailler pour être riche mais pour une liberté de tout instant. C'est hors de leur raison. Jamais ils ne pourront concevoir que la renommée n'ait rien à voir avec des sommes colossales d'argent. (Ibid.243)

Avec l'usage de l'anaphore "jamais », la narratrice montre que le comportement de sa mère est immuable, sa mère n'a pas changé d'un iota malgré la distance et le temps qui les séparent toutes les deux. Aucune ne cherche à faire de concessions, ni semble 
vouloir aller l'une vers l'autre. Malika est dans la résistance, elle refuse d'être comme sa mère, elle revendique sa liberté et elle sait qu'elle en a payé le prix fort, et donc, elle n'est pas disposée à céder du terrain à sa famille et surtout pas à sa mère. Elle adulte, en transformant la soumission réelle en pouvoir à travers l'écriture et l'imagination, elle parvient à se libérer de la souffrance générée par l'incompréhension des autres. Elle dira au sujet de sa mère, en évoquant son succès en France, en tant qu'écrivaine algérienne qui perce, ceci :

Et miracle, mon père permet à ma mère de répondre à une question face à la caméra :" quel effet ça te fait que ta fille soit devenue écrivain ? " La mine résignée, ma mère lève les bras au ciel : " qu'est-ce que tu veux que je te dise, mon fils, entre ma fille et moi, il y a toujours eu un livre". Même quand elle arrivait enfin à s'endormir, elle mettait son livre ouvert sur son visage! [...] c'est la plus belle phrase qui me soit jamais parvenue de cette bouche-là (Mokeddem, 2003 : 109.110) enfance qui «est toujours prise entre deux tensions antagonistes, la condition obligatoire de fille et celle » (Castellani, 2004 : 269) d'être en conflit avec sa mère :

Elle ne s'est même pas inquiétée de savoir pourquoi son aînée n'avait pas d'enfants! Qu'une femme puisse refuser d'enfanter est de l'ordre de l'inimaginable, de l'inconcevable pour elle. [...] Je n'ignore pas que mon anticonformisme ait pu rudoyer ma mère. Mais des brutalités, moi, j'en ai subi plus que mon dû. Cela m'a au moins appris à ne plus culpabiliser...L'éloignement ne remonte pas seulement à mon départ d'Algérie. Il vient de si loin. J'étais déjà l'enjeu de la rivalité entre elle et grand-mère. J'étais déjà dans la délivrance du seul fait de mon admiration de l'aïeule. J'ai mis le corps des textes entre elle et moi dès que j'ai appris à lire. (Mokeddem, 2003 :181.182)

La mère est dans le déni total, le lecteur constate cet état de fait au moment où la romancière remue sa vie, ou une partie, la plus importante nous semble-t-il en tout état de cause. Malika Mokeddem engage son moi dans l'écriture, elle retourne sur ellemême et elle tente d'expliquer sa souffrance dans sa famille, avec une mère qui a pour seule préoccupation le bien matériel que la narratrice pourrait apporter pour payer sa liberté. Dans le paragraphe précédent la romancière montre les difficultés de la mère à saisir son raisonnement et ses difficultés à elle pour lui expliquer ses convictions profondes sur l'enfantement, comme sur toutes les choses qui les opposent depuis très longtemps. L'écriture du moi chez Malika Mokeddem s'impose au lecteur comme "une écriture du dévoilement de l'intériorité. » (Miraux, 1996 : 26) Elle ne rêvait que de fuir cette misère et dans ses deux romans, la narratrice a vécu une enfance dans une fratrie nombreuse dans $L a$ Transe des insoumis, et réduite dans N'zid, à la figure du père et à quelques membres de sa famille qu'elle estime; et adulte, elle a vécu des aventures passionnelles; dont aucune ne l'a amenée à une vie de famille pleine et heureuse. Christiane Achour dit au sujet de la relation mère et fille que toute naissance à l'écriture et à l'individu-e » (Achour-Chaulet, $2007: 155)$ passe par « cette traversée pour une écrivaine » (155), elle illustre cela par une citation de Yvette Bénayoun-Szmidt et al, fort intéressante pour nous car elle corrobore ce que nous avons expliqué plus haut, le rôle de la mère dans la vie d'un auteur et comment Malika Mokeddem avait envisagé la place de la sienne même dans les fictions ou les livres qu'elle classe hors son autobiographie, l'absence de la mère, elle est complètement niée :

Je pense que la mère...elle est absente. Elle n'existe pas [...] j'ai mis du temps à m'en rendre compte. La mère n'est jamais là. Même dans N'zid, la mère a mis au monde une fille et elle est repartie en Algérie, carrément vers une autre terre. Je pense que 
c'est quelque chose d'inassouvi [...] Donc je ne peux l'inventer. (Bénayoun-Szmidt, $2007: 155)$

\section{L'image du père biologique et repères masculins}

Dans les romans de Malika Mokeddem, nous sommes en présence d'une femme en proie à des souffrances dues à sa famille et plus tard à son manque d'amour, et l'un des premiers traumatismes qui expliquent en grande partie son désarroi vient d'un souvenir d'enfance, un épisode qui l'a blessée, au moment où son frère est né, où le père a d'emblée montré sa préférence pour le garçon, c'est dans Mes Hommes, un autre roman autobiographique, qu'elle décrit la situation, qu'elle a repris dans la Transe des Insoumis et dans son roman, récit complètement éloigné de sa vie en apparence, Les Hommes qui marchent:

Mon père, mon premier homme, c'est par toi que j'ai appris à mesurer l'amour à l'aune des blessures et des manques. A partir de quel âge le ravage des mots? Je traque les images de la prime enfance. Des paroles ressurgissent, dessinent un passé noir et blanc. C'est très tôt. Trop tôt. Dès la sensation confuse d'avant la réflexion. Avant même que je sache m'exprimer. Quand le langage entreprend de saigner l'innocence. Du tranchant des mots, il incruste à jamais ses élancements. Après, dans la vie, on fait avec ou contre.(Mokeddem, $2005: 11$ )

Nous pouvons dire; pour comparer, que Malika Mokeddem tout comme Assia Djebar reprennent les événements marquants dans leurs romans, de façon pratiquement obsessionnelle, parce que les événements sont marquants, c'est par une plongée en soi, qu'elles restituent selon leurs visions les événements les plus malheureux, l'amour du père contrarié par les traditions et l'absence de l'amour maternel dans le cas de Malika Mokeddem. Dans le cas de Mokeddem, l'écriture était une urgence, une injonction pour retrouver sa liberté et se retrouver. Elle l'explique dans une des interviews qu'elle a données ce que représentait pour elle l'écriture et pourquoi, il fallait écrire; pour résumer ses propos voici juste un extrait qui nous semble important parce qu'elle l'a raconté presque ainsi dans le roman Mes Hommes, La Transe des insoumis : "L'écriture s'est accaparé ma vie pour y régner sans exclusive »(Lazhari, 2007 :33)

D'une manière plus symbolique, ce qu'elle appelle la métaphore de la perte, de l'absence et de la solitude, le lecteur les repère également dans le roman N'zid:

Nora pousse au bout de ses crayons. Entre les doléances des langues de l'enfance et le français qui la cueille dans la rue puis l'accueille à l'école, Nora n'a pas de terre. Elle n'en souffre pas. Bien au contraire. L'attachement à une patrie ne symbolise pour elle qu'un état de souffrance; celui de son père, celui de Zana, celui qui l'a privée d'une mère. [...] (173)

Dans notre analyse, nous constatons que la perte de repères familiaux se mue en un exil intérieur. Le père de la jeune narratrice, même dans La Transe des Insoumis n'est pas un père insensible, il est comme le père d'Assia Djebar contraint de suivre les traditions ancestrales dans un environnement hostile à toute émancipation des femmes. Ce n'est pas un père violent mais un père qui favorise la suprématie masculine, ce qui blesse la narratrice au plus profond, elle cherche une reconnaissance : à l'école, elle travaille bien dans l'espoir qu'il soit fier d'elle, tout comme Assia Djebar, elle va brandir devant son père ses notes, et la joie est à peine ressentie par les deux pères, l'un parce qu'il n'a pas apprécié le prix scolaire que sa fille a obtenu, l'autre, le père de Malika, n'est pas content, parce qu'elle n'est pas un garçon. Cette victoire sur le genre masculin ne 
compte pas. Dans ses relations avec les hommes qui ont traversé sa vie, elle le dit clairement dans une interview qu'elle ne « cherche pas un père » elle dit :

Je suis allée vers des hommes en les séparant justement diamétralement différents de lui. C'est-à- dire non autoritaires, non misogynes, libérés des conventions sociales, aimants... [...] Dès que m'apparaissait en eux quelque ressemblance rédhibitoire avec les travers du père, je m'enfuyais [...] D'avoir eu le culot de refuser tout ce que mon père a tenté de m'imposer des années durant, et finir par lui échapper, m'a rendue définitivement libre. Il m'était désormais impossible d'accepter le joug d'un autre homme (Lazhari, 2007 :57)

Cependant, l'image du père va évoluer à travers la narration. D'un livre à un autre, nous constatons que c'est l'image du patriarche qui change. Il passe d'une image du père intransigeant à celle du père compréhensif comme dans Les Hommes qui marchent, lorsqu'il devait prendre une décision quant à la poursuite des études de la petite Leila. Et l'image d'un père peu idéalisé, un père qui finit par se rallier à l'idée que, sa fille soit douée pour les études et qu'il fallait la laisser aller dans une autre ville. Le père biologique de la petite Leila incarne l'homme qui ne suit pas complètement les traditions.

Dans les autres romans où il est question de raconter des anecdotes avec le père, dans Mes Hommes et La Transe des insoumis et N'zid les pères sont représentés différemment. Ils sont plutôt vus comme des ennemis potentiels, des pères qui vont dévaloriser leurs filles et surtout les empêcher d'accéder au savoir. Dans l'ouvrage intitulé Malika Mokeddem Métissage de Christiane Chaulet Achour, la figure du père biologique est décrite ainsi :

Le père biologique, lui, est l'homme de la tradition qui ne prend pas d'initiative transgressive pour sa fille et auquel on doit toujours forcer la main pour qu'elle puisse poursuivre dans la différence (Achour-Chaulet, $2007: 123$ )

31 Le père qu'avait eu Malika Mokeddem est représenté dans les autres romans sous cet angle là, celui d'un père qui ne montre pas son affection et qui suit la mouvance imposée par la société même dans Les Hommes qui marchent, il avait fallu le supplier pour qu'il accepte de laisser la petite Leila continuer le collège puis le lycée; une situation des plus difficiles pour une fille de sud, habitant un petit hameau dont le décor de désolation aurait découragé les plus vaillants. Ses résultats scolaires sont brillants pourtant, ils ne vont pas impressionner outre mesure car c'est une fille qui les obtient. La narratrice vivra très mal cette ségrégation sexuelle dès son jeune âge au sein d'une famille conservatrice et c'est la nuit et au silence revenu dans la maison qu'elle prend le temps d'analyser la situation des dormeurs, les frères et les parents, son père qui fait la distinction nette entre les filles et les garçons : "image d'oubli, de fragilité et de mystère intriqués " et c'est dans ces moments de silence qu'elle découvre qu'elle aime malgré tout les siens. Malgré les différences infligées, elle devait trouver des stratégies pour échapper définitivement et ce sera la délivrance :

Il m'a fallu livrer une bataille sans nom et bénéficier de l'aide d'une étrangère, une française, la directrice de mon école, pour pouvoir franchir le seuil d lycée de la ville voisine. Le jour où je lui ai donné mon dossier de sixième à signer, pris de rage, mon père a roulé les feuillets en boule, les a jetés à l'autre bout de la pièce [...]

Avisée de ce refus, la directrice est venue le voir. (Mokeddem, 2003 : 117.118)

Dans cette citation, la narratrice sentait que ses études étaient son salut, elle savait que son père voulait au fond lui permettre d'étudier, cependant, il se faisait violence pour ne pas succomber à la pression sociale, la plaidoirie de son enseignante allait lui permettre d'aller ailleurs loin, non sans une certaine amertume. 


\section{Exil intérieur et exil géographique}

L'exil dans le cas de Malika « symbolise le drame d'une identité apatride et brisée (se trouvant entre les failles des deux cultures: française et maghrébine." (Mansueto, 2013: 36)Mais surtout, elle se sent abandonnée et écartée de sa famille. Pourtant, cet exil, elle l'assume vaille que vaille et en acceptant finalement «sa condition de déracinée " (Ibid. 36), peut-être qu'en créant un personnage féminin de Nora, de père européen, veut-elle inventer "d'autres identités fictionnelles» (Ibid. 36) pour occulter non pas son appartenance à la sphère maghrébine mais pour occulter une famille qui l'a poussée à l'exil.

Son bannissement débute dans son pays, durant les années de l'école. A l'internat, elle fait le même constat qui met la narratrice au pied du mur, elle s'interroge sur les différences qu'elle a avec ses petites protégées. Elle se demande pourquoi sa relation avec ses parents est si conflictuelle ? Pourquoi sent-elle le besoin de partir loin d'eux au lieu de vouloir rester à leur côté ? Elle est dubitative et elle pense à son propre destin et elle est persuadée d'avoir raison de vouloir partir.

La jeune fille va espacer ses visites à la maison, elle n'y va que pour remettre de l'argent à son père. Ce dernier l'a considère comme l'homme de la famille : « ma fille, maintenant tu es un homme! », cette affirmation ${ }^{10}$ consternante, pour elle aussi, permet néanmoins à la narratrice d'acquérir sa liberté, de fuir ce milieu familial pour très longtemps. Dans La transe des insoumis, il est question aussi de violences, la jeune narratrice voudra fuir les obligations sociales. Elle relate une agression à laquelle elle échappe in extrémis, le premier novembre. Un incident qu'elle répète dans Les Hommes qui marchent, Mes Hommes et $\mathrm{La}$ Transe des insoumis, tant l'événement était traumatisant, avec quelques variantes dans la description des scènes mais l'incident reste le même, avec la même force de violence :

Ce soir anniversaire du déclenchement de la révolution algérienne- comme cette locution me paraît hypocrite, ronflante ! - ils n'ont réussi à tuer en moi que des illusions sur les collectivités. [...] La marée blanche des haïks, les voiles des femmes, en occupe la moitié ${ }^{11}$. La foule sombre des hommes, l'autre. Ma sœur cadette et moi avons les seules têtes dévoilées. Nous venons à peine d'arriver que me parviennent des propos obscènes, des haleines chargées de vinasse. [...] J'endure leurs grossièretés en bouillonnant mais sans broncher car les ronchonnements des femmes alentour m'accusent de les exposer à la honte, à la vulgarité par mon impudence à me présenter là nue en pleine nuit. On dit nue parce que sans voile. [...] enhardi par les accusations des femmes, les incitations de copains aussi éméchés qu'excités, le plus âgé de la bande porte ses mains sur mes seins puis me pince une fesse. Je fais volte-face et dans un haut-le corps lui expédie une paire de gifles retentissante et un genou dans les couilles. [...] Ma riposte déclenche l'ire, le déchainement de la bande qui fonce sur moi, menaçant de me violer, de m'écarteler, m'éclater en morceaux, me... ${ }^{12}$ (Mokeddem, 2003 : 142.143)

La narratrice va subir une course poursuite d'une telle violence qu'elle conte au moins dans ses trois romans, avec de petites modifications mais les faits sont les mêmes. Elle risque sa peau à cause de ces jeunes gens qui ont voulu la violer et qui ont été presque encouragés par les femmes voilées, celles qui s'en sont prises aux deux jeunes filles qui ne portent pas le voile-protecteur que la jeune narratrice refuse de porter :

Prise de panique, je saisis la main de ma sœur et m'élance à travers la place en direction de l'angle où doivent se tenir mon père et mon oncle ; un sprint effréné, 
poursuivi par la horde en rage, durant lequel je reçois toutes sortes de projectiles, de coups d'injures. Deux voix cependant surplombent les invectives de cette lugubre soirée. Celle du photographe Bellal: «Malika, par là, par là, vite!» sa boutique est ouverte [...] Nous nous engouffrons dans son magasin ma mère et moi. Il a à peine le temps de descendre son rideau de fer que la foule est déjà dessus à essayer de le défoncer. (Ibid. 143.144) dans son discours elle avoue avoir donné plus de détails dans Les Hommes qui marchent à la page $144 \mathrm{du}$ roman La transe des insoumis. La romancière dans son écriture personnelle a réélaboré ses souvenirs les plus traumatisants et celui du $1^{\text {er }}$ novembre est l'un des souvenirs les plus choquants et plus révoltants aussi, pour elle, en vérité, les événements autobiographiques que proposent Malika Mokeddem, comme tous les autobiographes d'ailleurs, sont ceux qui ne retiennent que «les grandes lignes ${ }^{13}$ d'une $^{2}$ existence, en négligeant les éléments non concordants ou subalternes » (Hubier, 2003: 76) et comme Assia Djebar, elle a opéré des choix conscients ou inconscients des événements de son vécu.

Elle va néanmoins vaincre les siens le jour de la commémoration de l'indépendance, le premier novembre lorsqu'elle a failli se faire violer et lyncher par une horde de pervers. Sa réputation était faite, elle n'aura aucun mal à éloigner le danger du mariage. Elle savoure sa nouvelle fonction, puisqu'elle n'est plus obligée de rentrer chez elle : " je suis parvenue à m'arracher au corps familial » (Mokeddem, 2003 : 129)

Le père de la narratrice dans le roman La Transe des insoumis n'est pas forcément contre sa fille, puisqu'il va tenter d'ignorer les rumeurs contre sa fille, elle reprend cette image du père qui malgré sa naissance non désirée, elle va acheter son silence et sa liberté à la fois :

Depuis cette mort et parce que je sais que maintenant qu'aucun joug ne pourra m'empêcher d'aller à la faculté, je ne dis rien à personne. [...] Je n'ai plus envie de voir ma mère tressaillir, se cabrer comme si le ciel allait se casser sur sa tête dès que j'ouvre la bouche. J'ai définitivement quitté son rang. [...] Salaire après salaire, j'ai acheté ma liberté. Comme une esclave. Ma liberté et ma solitude. Les deux vont ensemble. Pour moi, elles ont grandi ensemble dans cet exil magnifique, le savoir. (Mokeddem, 2003 : 158)

$\mathrm{Au}$ fond d'elle-même, elle sait que ce qui la sépare de son père n'est pas le manque d'amour mais c'est la tradition, elle le met bien en évidence à la fin du roman La Transe des insoumis en des termes plus ou moins indulgents, et on ne saurait dire si c'est dû au fait que c'est à la fin de la vie de son père ou c'est parce qu'elle l'a réellement aimé :

Je distribue les présents et retourne m'asseoir près de mon père. Lui, il cherche aussitôt ma main, la presse. Je me calme. [...] C'est pour lui que je suis revenue. Pour que cet amour, encore vivant mais jusqu'alors anéanti, confisqué par la tradition, les conventions sociales, mes rébellions, puisse enfin se manifester un peu. [...]J'ai seulement tenu à exister entièrement à ses yeux comme aux miens. Maintenant mon père est au bout de sa vie. Je ne veux pas qu'il parte avec cette souffrance. (Ibid. 243)

La représentation du père n'est pas toujours la même, parce que celle représentée dans les autres romans va changer, et le moins qu'on puisse dire c'est que le père de Nora dans N'zid est un père aimant qui aurait aimé protéger sa fille et la voir évoluer dans une vie normale en famille avec sa mère et son père. Il va donc l'élever parce que sa mère ne pouvait assumer cette tâche, elle est morte dans des circonstances tragiques. Cependant, selon Christiane Achour, Samuel, l'Irlandais, pour Nora dans N'zid est un 
père qui remplit tant bien que mal son rôle, parce que de doute façon, il va disparaitre et laisser la jeune fille seule au monde.

Ainsi à travers les interrogations de la jeune Nora sur ses origines, nous pouvons dire que Malika Mokeddem construit son identité à travers « des repères masculins "(AchourChaulet, $2007: 125$ ), l'amnésie du personnage de Nora va susciter des questionnements sur ses origines et par ricochet sur son identité, de ce fait l'animosité réellement vécue et la création d'une image du père va déclencher le processus de l'exil intérieur d'abord puis l'exil géographique, et elle se retrouve de plain-pied dans le processus du métissage parce que fille du désert va changer d'univers et devient malgré elle, celle qui va changer d'espace géographique pour aller vivre en méditerranée, donc à son réveil Nora se rend compte de son amnésie et examine la situation dans laquelle elle se retrouve :

Assaillie par des intuitions fugaces ou troublantes, le nez collé à la carte, le souffle haletant et le regard dérivant sur le pourtour de la mer, elle prend conscience du rocambolesque de sa quête : [...] si elle pouvait se donner un pays, lequel choisiraitelle ? Un petit rond, tracé au crayon, sur la côte algérienne attire son attention. L'Algérie ? L'Egypte ? Israël ? Elle stoppe là l'énoncé des noms de pays et tressaille. Pourquoi cette question se dérobe-t-elle? [...]Tous ceux qui vivent entre revendications et ruptures. Avec ce petit rire de dérision, elle se dit : " Ils n'ont qu'à aller à la mer comme moi, comme toutes les épaves. La mer est douce pour les épaves "(Mokeddem, 2001 :21.22)

Christiane Achour explique parfaitement comment "l'angle d'étude du masculin» (Achour- Chaulet, 2007 : 126)certifie qu'avec N'zid «il faut passer du désert réel, au désert métaphore de l'écriture possible pour que s'accomplisse le miracle d'une origine reconstruite[...] il est métaphore de l'imaginaire et impulsion essentiel de la libération » (Ibid. 127) en sachant que dans tous ses romans, la libération s'est faite dans la douleur et ce sont : « Les pères et les mères de substitution qui aident à avancer, aident à se reconstruire mais ne comblent pas la carence d'enfance. [...]Les héroïnes de Malika Mokeddem les cherchent ailleurs "(Ibid. 127)

Avec N'zid, c'est sur la mer que le lecteur retrouve la jeune femme. Elle apprend justement qu'elle est la fille d'une Algérienne Aïcha et d'un Irlandais, Samuel, cette découverte la force à faire une introspection et elle se parle très souvent d'ailleurs, nous le voyons à chaque paragraphe mis en italique dans le texte. Au fil de la narration, Nora recouvre sa mémoire partiellement et deux initiales vont être données :

La voix s'esclaffe et la poursuit. Est-ce celle de son père? De J ? Elle se tient aux haubans pour ne pas tomber à la renverse, terrassée par le désespoir, et crie :

-J.J.J ? Jamil ? Jean ? Joan ? Joachim ?» (Mokeddem, 2001 : 17)

Jamil est un prénom que l'on retrouve dans beaucoup de ses écrits. Jamil est le jeune homme qui va tomber amoureux de Leila, il est aussi dans L'Interdite et Mes Hommes ainsi que dans N'zid, il est dans tous les cas en compétition avec soit le français Jean-Louis, soit Vincent et jean dans N'Zid. Jamil est l'homme qu'elle désire retrouver mais elle apprend à la fin qu'il est mort en Algérie ainsi que Jean qui a rejoint les rangs des intégristes. Christiane Achour explique ainsi :

Le retour de la dernière scène de violence qui avait provoqué l'amnésie est l'ultime maîtrise de la mémoire. C'est à re-vivre, "N'zid ", à recommencer que Nora doit s'acharner une nouvelle fois avec, à l'horizon, l'intérêt amoureux de Loïc et

l'affection maternelle de Zana. (Achour-Chaulet, 2007 : 129)

Nous allons voir que les personnages masculins qui semblent être laissés en second plan, occupent au contraire une place nécessaire dans ce que nous pourrions 
considérer comme une justification à la grande solitude que la femme-narratriceécrivaine a vécue. Ce qui est remarquable c'est que l'un des thèmes fondateurs obsessionnels de l'univers romanesque de Mokeddem est cette présence/ absence des autres femmes mais de la présence constante des hommes qu'elle a aimés et haïs à la fois, Christiane Achour avance à juste titre en disant :

Si la solitude de la femme qui entend vivre sa vie- c'est sans nulle doute, une des thématiques essentielles de cet univers romanesque- est dominante et obsessionnelle, cela n'empêche pas la voix narratrice de camper des «frères " qui partagent cette même recherche. [...] Il y a d'autres femmes auxquelles on peut se confier d'autres femmes relais ou repoussoirs mais le dialogue de fond se fait au féminin singulier/masculin pluriel. [...] Ainsi, de fiction en fiction, l'écriture romanesque de Malika Mokeddem s'affirme comme interrogation des rapports entre les sexes (Ibid.130)

Nous pouvons dire que la narratrice- personnage qui parle à la première personne, est en perpétuel combat contre les siens et que même ses amours semblent tout aussi contrariées, ce qui accentue la solitude parce qu'elle refuse de suivre l'ordre établi par la société des hommes et des femmes qui se sont liguées contre elle longuement.

\section{Les amours contrariées}

41 Après son père et les différents personnages qui incarnent la figure du père, qui comme le signale Christiane Achour sont parfois «à la hauteur ou pas dans la vie de leurs filles, ils finissent toujours par disparaitre ${ }^{14} »$ (Ibid.124), d'autres hommes vont se succéder dans l'existence de la narratrice, ses nombreuses conquêtes qui ont réveillé en elle des sentiments et qui parfois ont accentué le sentiment d'être différente, d'être une étrangère dans son propre pays. Malika Mokeddem va raconter dans plusieurs de ses romans comment les hommes de sa tribu l'ont traitée, parfois c'est une romancière désabusée qui met en évidence une image masculine pas toujours reluisante et qui aura tôt fait d'accélérer son départ vers d'autres cieux, d'autres espaces et c'est précisément lors de son exil forcé qu'elle connaitra véritablement le grand amour. Jean- Louis connu à Paris, il deviendra vite indispensable à la vie de la narratrice.

Amoureux l'un de l'autre, ils ne se quittent plus. Il lui fera découvrir les senteurs, le gout de la mer, la cuisine, et un engouement pour la mer. Ils resteront ensemble 17 ans: «J'ignore que nous resterons dix-sept ans» (Mokeddem, 2005 : 73) une anticipation qui suppose une séparation, en effet, ils se séparent indirectement d'une petite liaison, avec la sœur de la narratrice, venue se réfugier chez eux, et qui a brisé un lien qui semblait indéfectible et indirectement à cause de l'écriture. Elle ne parlera pas de cette liaison dans La Transe des insoumis.

Dans ses romans, elle dit qu'elle est pourtant encouragée par Jean-Louis; il se sentira de trop dans son univers livresque. Le succès de ses romans, qui pourtant, la délivrera de ses angoisses ne parviendra pas à sauver son mariage. Dans cette partie de cette analyse, il ne faut pas voir seulement dans le genre autobiographique une forme d'exhibitionnisme ou d'indécence à montrer mais une envie de "regarder longuement la figure recomposée par l'acte d'écrire " (Miraux, 2003: 36) Michel Leiris évoque l'idée d' " architecturer le moi pour en tirer de nouvelles perspectives » (36), Malika Mokeddem dans le récit qu'elle fait de cette relation amoureuse donne une certaine authenticité de ses sentiments intimes, ce qui lui permet également d'approfondir la connaissance de soi. 
Elle débutera son roman par cette métaphore du lit, qui fait immédiatement référence à son mari et de la vie qu'elle a partagé avec lui :

Il ne dormira plus avec moi dans ce lit. Je suis encore anesthésiée par la brutalité de cette certitude. Je suis comme un amputé au réveil d'une opération. Quand la douleur est encore absente. Elle viendra lorsque l'absence aura pris corps. Avec la pleine conscience de la mutilation. (Mokeddem, $2003: 15$ )

Ce n'est pas le vécu en général que Malika Mokeddem a remanié mais elle a choisi un moment bien particulier celui de la séparation qu'elle a vécue de manière brutale, le champ lexical du médical montre justement que la rupture est vécue comme un dommage psychologique plus que physique. Pour nous, il ne s'agit pas de l'expliquer sur le plan psychanalytique, nous ne saurions le dire avec précision, mais de montrer comment l'écriture autobiographique a rendu compte du sens des sentiments et donne un sens au moi profond de la romancière. Elle parle de membre « amputé, de mutilation, et de douleur ", l'absence de douleur physique n'exclut pas la présence de la douleur d'un tout autre ordre. Tout son univers s'effondre, on est dans la fracture où le bonheur est perdu. Nous sommes bien dans cette catégorie d'écrivains qui sont en quête d'un bonheur perdu, Malika Mokeddem reproduit dans ces romans, avant la séparation l'image d'une « vie ouverte à tous les possibles (Miraux, 2005 :38) mais aussi «certainement des illusions multiples, (qui) constituent des instants regrettés d'allégresse "( Miraux, 2005: 38)elle va reprendre dans $L a$ Transe des insoumis ce que Jean-Louis avait été pour elle, malgré quelques épisodes négatifs, elle relate des périodes heureuses: "alors que le présent de l'énonciation, le présent de l'écriture, se situe dans une période dégradée et malheureuse » (Ibid. 38) de sa vie:

Le compagnon que je me suis choisi est français. S'il n'a rien du macho, loin s'en faut, c'est parce qu'il s'en est toujours défendu comme d'une forme d'infirmité. Seulement, il panique à me regarder m'éloigner dans l'écriture. Il craint de me perdre et me perd pourtant. J'ai longtemps espéré qu'il ne me lâche pas la main. Au fil des années, ses baisers dans le demi-sommeil ont fini par me faire croire notre amour éternel. A l'effleurement de ses lèvres sur ma peau, la nuit, ses bras et son corps étaient mon continent. (Mokeddem, 2003 : 24.25)

La rupture n'a pas empêché la romancière de garder une certaine amertume, parce qu'elle a été aussi dure si ce n'est aussi pénible que sa séparation des siens ; la solitude est son quotidien désormais et elle a conscience du prix de la liberté, c'est l'écriture qui sera à la fois son échappatoire et c'est aussi ce qui a mis en branle sa vie conjugale qu'elle croyait éternelle. Du moins espérait-elle que Jean-Louis puisse faire abstraction de toutes les différences et rester avec elle, lui apporter son soutien et contredire la pensée générale sur la gent masculine dont parle Marguerite Duras et que la romancière reprend dans son roman, quand elle dit «Les hommes ne le supportent pas : une femme qui écrit [...] » (Mokeddem, 2003 : 25)

Elle parlera de Jean-Louis en deux endroits du texte, mais dans le chapitre «l'homme des traversées ", elle lui consacre une grande part de son roman et dans d'autres romans car son absence et le lit conjugal rappellent sans cesse la douleur de l'absence. Elle restera avec lui, mais son succès désespère son mari : "dès que tu t'es mise à écrire, j'ai compris que tu partais sans retour en me laissant sur un quai » (Mokeddem, 2005 : 135)

Jean-Louis est pourtant l'homme, le mari qui a su faire en sorte que la France devienne son deuxième pays. Dans son nouveau lit, trop grand pour contenir un petit bout de femme, elle se sent seule, et abandonnée. Néanmoins, elle désire vivre un amour charnel, la passion qui l'habite est celle d'une femme venue d'ailleurs, d'un milieu de 
tous les interdits. Les nuits sans les bras et un corps qui « creuseraient » à ses manques sont insupportables sans Jean-Louis, c'est le seul homme de tous ceux qu'elle a connu qui a pu «l'apprivoiser », dans cet état d'abandon, elle ne veut plus et ne peux plus vivre d'aventures passagères.

L'écriture va l'aider à supporter ce manque, en se soumettant strictement aux règles de l'autofiction, mise ne abyme ; l'écriture est pour elle très importante :

Un soir où Jean-Louis et moi, nous nous parlons enfin, vraiment, il me suggère : "Ca fait des années que tu dis que tu vas te mettre à écrire. Fais-le ! Maintenant! " Cette observation tourne dans ma tête. Elle ne m'a pas quitté un instant [...] J'écris [...] et de constater à quel point l'écriture m'emporte. (Mokeddem, 2005 : 132)

Elle écrit partout chez elle à Montpellier ou en mer, c'est d'ailleurs en cet endroit qu'elle a été la plus inspirée : «Par beau temps, en mer, j'écris [...] l'un des textes écrits en cours de navigation : la mer, l'autre désert »(134.135). La narratrice insiste sur la nécessité d'écrire, l'anaphore du verbe écrire en est la preuve, la modalité met en évidence un impératif primaire de dire les choses et être dans la position d'attaque pour dénoncer les exigences sociales injustes et perçues comme telles :

Il faut écrire cette lutte. Les nichées de lits de la cité, leur cocasserie, leurs acquis. Leurs épopées parfois belles, souvent décevantes. Il faut écrire les hordes de flics qui nous traquent le soir dans la ville.[...]. Ecrire nos affrontements dans les commissariats miteux d'Oran [...]. Ecrire les dérives, les aberrations du système Boumediene. Ecrire les gardes de service de maternité. Les accouchements à la chaine, leurs scènes felliniennes. (Mokeddem, $2003: 191.192)$

Les rencontres avec les hommes qui ont traversé sa vie, ne se sont pas faites par hasard, pour elle, le hasard n'est que «le fruit, parfois masqué, inconscient de nos aspirations » (Mokeddem,2005 : 198) Néanmoins, les rencontres rêvées finissent toujours par la faire fuir, le trauma subi par les nombreuses ruptures et surtout avec son mari, montre qu'elle a "peur d'aimer» (211) Le dernier chapitre du récit La Transe des insoumis, semble être une synthèse de tout ce pour quoi elle croit ; la solitude, l'amour, mais surtout "l'amour d'elle-même", sa solitude n'est pas pesante, mais elle est "imbriquée ", "elle (la solitude) s'érige en héroïsme des mal-aimés» (214) Ce chapitre est une longue réflexion sur son vécu, sur son passé, présent, elle projette un futur où la légèreté serait sa nouvelle devise.

Pour élucider le parcours un peu chaotique de la romancière, le lecteur peut constater qu'elle est désireuse de se comprendre et de s'expliquer, ainsi elle rend compte de toutes les histoires amoureuses qu'elle a vécues et les ponctuant de "ces sortes de commentaires extrêmement instructifs qui permettent de comprendre les motivations profondes » (Miraux, $2005: 30$ ) de son moi profond.

Parmi les amours contrariées de la narratrice-personnage qu'elle reprend néanmoins dans d'autres romans est son amour pour le Kabyle qu'elle a connu durant ses études de médecine à Oran, et dans N'zid, elle y fait allusion d'une manière métaphorique, une relation antérieure à sa rencontre avec Jean-Louis. Elle reprend l'histoire d'amour qu'elle avait eue avec cet homme, blond, beau aux yeux clairs, qu'elle croyait suffisamment amoureux d'elle pour braver toutes les conventions sociales. Cet homme a cédé aux pressions et ne l'a pas épousée finalement. Cet épisode de sa vie est vécu par beaucoup de ses personnages féminins, Selma de L'interdite et Des Rêves et des assassins, Mes Hommes ${ }^{15}$, et dans le roman de N'zid, c'est un personnage marin, la méduse qui va représenter les protagonistes féminins qui la représentent : 
Puis elle abandonne ses doigts à la transe des couleurs et peint l'histoire d'une méduse amoureuse d'un oursin. Un sédentaire des plus barricadés qui ne la regarde même pas. Vissé à son rocher, au milieu d'une tribu hérissée, il ignore totalement la subtilité des reflets de sa peau diaphane. Il s'en fallu de peu que son ballet de séduction, autour de lui, ne tourne en danse macabre. Elle a failli se déchiqueter sur les piques de la communauté. Elle s'éloigne à regret. Dans sa fuite éperdue, elle rencontre une baleine à qui elle raconte ses déboires : " tu comprends, je suis trop transparente! Il ne pouvait pas me voir. Je n'existe pas pour lui ", conclut la pauvresse. "Je te vois bien, moi, qui suis un poids lourd. Pauvre cloche, tu es tombée sur un charbon. Un de ces demeurés d'immobiles seulement préoccupés à se momifier les racines. Ces espèces-là prolifèrent en ce moment. Ils s'abêtissent par bans à force de se fixer les crampons. [...] Je veux te montrer les nomades des surfaces et des profondeurs. Ça va t'ouvrir les horizons "(Mokeddem, 2001 :34.35)

Le même événement est relaté dans La Transe des insoumis peut-être d'une manière moins poétique ou moins métaphorique, elle raconte ses amours de jeunesse quand elle était à l'université, le détail de cette relation est raconté dans Mes hommes où la narratrice raconte comment ce jeune homme l'avait attiré, et comment elle a fini par succomber à son charme :

Depuis quelques temps il en est un! Un qui ne me conviendrait pas. [...]C'est pour celui-là que je craque. C'est un désir violent, envoutant. Je me dis : "c'est peut-être ça l'amour. Ce tremblement inconnu. Cette ... " Puis m'ébroue: " Arrête, arrête les niaiseries, c'est pas pour toi ! " J'ai beau essayer de me moquer de la chimie des émotions, ça ne prend pas. La douce euphorie des toquades chamboule mes repères. [...] Il se dit qu'il est le fils de sa mère- une mère de Nédroma, l'un des bastions de la tradition- qu'il finira marié par elle comme la plupart des copains. [...] Pour tous les actes qui engagent leur vie, ils regagnent le bercail conservateur... (Mokeddem, $2003: 175.176)$

Ce personnage est repris dans un de ses autres romans, Les rêves et les Assassins, où elle va retrouver la nostalgie de ce bourgeois qu'elle a aimé et qu'il a regretté de ne pas l'avoir épousée. Les amours contrariées, c'est aussi la partie réservée à « cette rumination successive » qui lui a tant fait mal. C'est l'histoire des amours empêchées en Algérie :

Il faut écrire cette lutte. [...] Il faut écrire les hordes de flics qui nous traquent le soir dans la ville. [...] Ecrire nos affrontements dans les commissariats miteux d'Oran. Parfois jusqu'à 2 heures du matin. Une guerre des nerfs. [...] cependant l'étouffement, les abus, l'arbitraire, l'instinct du danger, la répétition des impossibilités, des faillites de l'amour finiront par me faire détaler. (Mokeddem, $2003: 189.192)$

La narratrice médite sur l'amour en général puis analyse sa propre situation: son amour (pour Jean-Louis) «s'est laissé endormir par l'habitude " (Mokeddem, 2003: 111) Et ou «par devoir» (Mokeddem, 2003 : 111). Dans cette état de raisonnement, ses nuits sont peuplées de rêves, et elle dévoile un aspect de son intimité ; des rêves érotiques ce qu'elle appelle "toutes les soifs de ma nuit », elle a la rage au ventre, et l'abstinence est pour elle un malheur. Le rêve offre à la jeune femme la possibilité de fuir sa solitude et son exil intérieur. La trajectoire, le tracé de vie de cette auteure semble être un tracé discontinu animé par des bribes de souvenirs comme l'a signalé Najiba Regaïeg: "l'écriture fait ressac », ce qui veut dire un retour vers l'enfance et l'adolescence, des âges de tous les rêves.

Le classement établi par les spécialistes de l'écriture autobiographique, et selon les critères qu'ils ont établis ; ces derniers ne semblent pas s'appliquer à tous les écrivains, nous avons un contre-exemple: Malika Mokeddem. Elle n'a pas écrit en tenant compte de ces critères : la religion par exemple ; l'aurait empêchée de raconter certains détails 
que nous n'avons pas pu tous les développer dans cette présentation. Et justement parce qu'elle est une femme, elle n'a pas eu des scrupules à montrer sa mère sous un angle très peu reluisant, en faisant ressortir les travers d'une vie familiale et montrer que la vie sociale algérienne est rétrograde et étouffante pour une femme qui aspire à la liberté. Cette liberté l'a d'ailleurs poussée à chercher ailleurs son bonheur, dans un autre pays avec d'autres personnes.

Malika Mokeddem aura senti à un moment de son existence, un besoin vital d'écrire et de noircir des pages pour raconter tout ce qui a bouleversé sa vie et ce qui va apporter au lecteur l'explication de sa vocation de future écrivaine; des moments de lecture délicieux dans une bibliothèque rudimentaire, des jugements sévères de sa mère mais des encouragements de certains de ses proches notamment son mari et certains de ses amis. A travers son écriture, elle tentera, à partir des figures féminines d'aller sur les traces de son passé qui ont fait d'elle ce qu'elle est devenue. Elle essayera de comprendre, à notre sens que toute son existence est ce qu'elle est par l'influence de ces deux personnages liés au sacrifice, sa grand-mère, conteuse qui l'a inspirée, et son mari qui a dû se séparer d'elle pour la laisser poursuivre son rêve. Dans le cas de Malika Mokeddem l'écriture intimiste rime donc avec les révélations fracassantes, elle ose l'indicible dans une littérature maghrébine qui tend à être dans la réserve dans beaucoup de cas, parce que pour elle «seuls comptent dans ce cas le contexte et le souci de vérité $[. .]$.$» (Mokeddem, 2003: 12$ )

\section{BIBLIOGRAPHIE}

CHAULET- ACHOUR, Christiane. Malika Mokeddem, Métissages. Editions du Tell, 2007.178P.

GASPARINI, Philippe. EST-IL JE ? Roman autobiographique et autofiction. Seuil, 2004. (Col. Poétique). 393P.

HUBIER, Sébastien. Littératures intimes. Les expressions du moi, de l'autobiographie à l'autofiction. Armand Colin, 2003 160P.

LEJEUNE, Philippe. Le Pacte autobiographique. Paris, du Seuil, 1975. 357P.

LEJEUNE Philippe. Les Brouillons de soi. Editions du Seuil, 1998, (Col. Poétique) 426P.

MIRAUX, Jean-Philippe., L'Autobiographie, écriture de soi et sincérité. Paris, Editions Nathan, 1996. (Col.128 Lettres) 1996. 128P.

MOKEDDEM, Malika. L'Interdite, Editions Grasset et Fasquelles, 1995. (Col. Le Livre de poche $\left.\mathrm{n}{ }^{\circ} 13768\right) .180 \mathrm{P}$.

MOKEDDEM Malika. N'zid, Editions du Seuil, 2001.213P

MOKEDDEM Malika. La transe des insoumis, Editions Grasset et Fasquelle, 2003 (Col. Le Livre de poche $\mathrm{n} \circ 30437)$. 254P.

MOKEDDEM, Malika. Mes hommes, Editions Grasset et Fasquelle, 2005 (Col. Le Livre de poche $\mathrm{n} \circ 30848) .218 \mathrm{P}$. 
MOKEDDEM, Malika. Je dois tout à ton oubli, Editions Grasset et Fasquelle. 2008 (Col. Le Livre de poche $\mathrm{n} \circ 32125)$. 160P

BLANCHAUD, Corinne. «L'écriture romanesque de Malika Mokeddem : autobiographie, témoignage et fiction » dans Autobiographie en situation d'interculturalité, coordonatrice Bererhi Afifa, Editions du Tell, 2004. 554P Pp 311.330.

BOURICHE, Soraya.. « Malika Mokeddem, La désirante » in Au fil des pages. Nov. Déc. 2011 :L'ivrEscq $1^{\text {er }}$ Magazine littéraire. $\mathrm{N} \circ 14$

DETREZ, Christine. «A corps et à cri ; résistances corporelles chez les écrivaines maghrébines » dans Ecriture féminine : réception, discours et représentations. Oran, Éditions CRASC, 2010, ISBN : 978-9961-813-39-3.284P Pp69.79.

CASTELLANI, Jean-Pierre. « Les figures de la mère et de l'enfance dans « Denier du rêve » et « Rendre à César » de Marguerite Yourcenar » dans L'autobiographie ensituation d'interculturalité, sous la direction de Bererhi Afifa., 2004, Editions du Tell. Pp 269. 286.

LAZHARI, Labter. Malika Mokeddem, à part entière. Entretien avec Malika Mokeddem, Editions SEDIA, 2007. 72P

MANSUETO, Claudia « La problématique de l'exil dans la littérature maghrébine féminine : Garçon manqué de Nina Bouraoui, Ce pays dont je meurs de Fawzia Zouari et Zeïda de nulle part de Leila Houari », No 4. Mai 2013, Pp. 34.45. « disponible sur » Les Cahiers du GRELCEF.(consulté le 10 juin 2019). www.uwo.ca/french/grelcef/cahiers_intro.htm

\section{NOTES}

1. Une critique littéraire sans doute, citée par Sébastien Hubier. Elle était un écrivain américain de romans, de poèmes, d'histoires, d'essais et de biographies. Son autobiographie: une étude critique et comparative (1909), était le premier livre sur le sujet.

2. MAY Georges est cité par Hubier Sébastien dans son ouvrage.

3. Malika Mokeddem médecin exerçant en France, descendante de nomades est née le 5 octobre 1949 à Kenadsa (Saoura) dans l'Ouest du désert algérien. Elle est auteur de multiples romans, et comme elle ses personnages féminins, ses héroïnes ne manquent pas de détermination et de décisions. Les Hommes qui marchenté dité en 1990. Le Siècle des sauterelles, chez Ramsay, En 1993, elle publie son troisième roman, l'Interdite chez Grasset. En 1995, parait son quatrième roman, intitulé Des Rêves et des assassins, En moins de 10 années, elle publie quatre nouveaux romans, 1998, La Nuit de la lézarde, en 2001, édition Seuil, N’Zid, un autre récit autobiographique, en 2003, La transe des Insoumis, Mes Hommes, en 2005 est un récit personnel comportant des tranches de vie déjà racontées dans pratiquement tous ses romans.

4. N'zid est un roman de Malika Mokeddem.

5. Ce passage mis en italique est mis ainsi dans le texte, nous pensons que l'auteure fait de l'autocitation, elle ne l'indique pas par une notre de bas de page mais le met par la typographie particulière et par l'usage des guillemets.

6. Misologue : celui qui n'aime pas le langage.

7. Le lit partagé avec ses frères et sœurs. Elle sent le poids des couvertures en laine sur son corps frêle

8. Nous laissons le passage en italique parce qu'il est inscrit ainsi dans le roman, il s'agit des réflexions, une sorte de voix intérieure qui commente les événements. Et dans ce cas, il s'agit de la mère de Nora, mais l'image semble être celle de la romancière. 
9. Zana ressemble au personnage de la grand-mère de la romancière qui rappelons-le ici, qu'elle a été un modèle pour la romancière et les personnages féminins qui la représentent, ont connu une grand-mère ou une vieille femme conteuse d'histoires.

10. C'est un fait relaté dans plusieurs romans: Les hommes qui marchent, Mes Hommes, L'interdite, Des rêves et des assassins. A chaque fois, avec quelques variations, elle remet sur le papier le fait que le père ne la considère pas comme une fille mais comme celle qui rapporte de l'argent et donc comme un homme, elle reçoit cette piètre considération.

11. Place immense carrée dans la ville.

12. Voici la version du même fait dans le roman Mes hommes à la page 103. Mais pas complète : «c'est en 1965. [...] Un soir de $1^{\text {er }}$ novembre, il décide de rester ouvert jusqu'au feu d'artifice prévu sur la grande place pour la commémoration du déclenchement de la guerre de la guerre d'indépendance. Il veut faire des photos des illuminations et de la liesse populaire. Hélas pour lui et pour moi, il ne fera aucune photo ce soir-là. Ce soir- là, il baisse son rideau de fer dans mon dos. Face aux gueules tordues par la violence de centaines d'hommes qui m'auraient lynchée sans son intervention in extremis, seulement parce que je n'étais pas voilée. [...]

13. Selon G. Gusdorf cité par Sébastien Hubier dans son ouvrage «Les écritures du moi exposent le moi d'une manière privilégiée, mais nullement directe et totale. Aucun journal, aucune autobiographie ne peut être considérée comme l'énoncé littéral de la vie dont il porte témoignage. P 74.

14. Le père de Sultana et le père de Nora ont joué un rôle plus ou moins bien dans leurs vies. La vie des deux jeunes filles.

15. Elle a intitulé le chapitre dans le roman Mes Hommes qu'elle a consacré à son amour de faculté «Le goût du blond» voilà ce qu'elle dira de lui : "Je sors des locaux de la faculté de médecine lorsque je vois Saïd pour la première fois. Il a des cheveux et des yeux clairs [...] j'emporte avec moi l'image de sa blondeur [... ] c'est un fils de bourge [...] Saïd est Kabyle, de taille moyenne. Les parents de Saïd ne veulent pas de moi [...].. Saïd Pour les infléchir, il me jure qu'il ne reviendra chez lui qu'à leur reddition. [...] Saïd, lui, c'est certain, ne fera jamais rien sans l'accord des siens. [...] et l'image ultra- conservatrice de la tribu de Saïd est un tel repoussoir. [...] Saïd a fini par regagner le bercail de ses traditions.

\section{RÉSUMÉS}

Des études universitaires qui traitent les textes autobiographiques posent la problématique suivante comment racontent les écrivains leurs vécus. La question de l'écriture de l'intime reste posée parce qu'il y a des critères qui peuvent expliquer la proportion des révélations intimes dans un récit de vie. Les chercheurs ont donc établi un classement des écrits intimes pas toujours évidents à appliquer aux écrits autobiographiques. Les critères tels que la religion, la nationalité, le sexe, la situation sociale ou économique des différents auteurs » (Hubier, 2003 : 68) ont montré qu'il est assez compliqué de les voir appliqués sur tous les textes, cela tout simplement dire que les écrivains autobiographes n'écrivent pas leur vie intime de la même façon. Nous avons tenté à travers l'exemple de Malika Mokeddem qui est une écrivaine algérienne d'expression française de montrer justement qu'elle fait exception par rapport à d'autres écrivains maghrébins qui eux optent pour une certaine réserve voire ont tendance à embellir leur vie familiale et sociale. Beaucoup des critères cités ci-dessus, ne s'appliquent pas sur elle, elle fait des révélations sur son vécu sans tenir compte par exemple du facteur religieux, ou social. Au contraire, elle dit sans 
tabous des vérités qui pourraient heurter la sensibilité des siens, mais elle ne tient pas compte de ce facteur, parce que ce qui importe pour elle c'est dire sa vie tumultueuse avec les siens qui l'ont poussé à l'exil. Elle fait un plaidoyer pour justifier un peu son refus de s'identifier à sa communauté. Chaque étape de sa vie, l'éloigne un peu plus des siens et même des personnes qui l'ont aimée.

University studies dealing with autobiographical texts raise the following problem : How writers tell their stories? The question of intimacy writing remains because of criteria that can explain the proportion of intimate revelations in a life story. Therefore, researchers have established a classification of intimate writings which is not always obvious to apply to autobiographical writings. Criteria such as religion, nationality, gender, social or economic status of the various authors (Hubier, $2003: 68$ ) have shown that it is somehow complicated to apply them to all texts. It means that autobiographical writers do not write their intimate lives in the same way. We have tried through the example of Malika Mokeddem, who is an Algerian French-speaking writer, to show precisely that she is an exception compared to other North African writers who opt for a kind of reserve tendency to make their family and social life more beautiful. Many of the criteria cited above are not applicable to her, she makes revelations about her experience without considering, for example, the religious, or social factor. She says, instead, and without any taboo the truth even if it could sometimes offend the sensibilities of others. Malika Mokeddem does not consider this factor because, the most important thing for her is to relate her tumultuous life with her family and people who drove her to exile. She makes a plea to justify her refusal to be identified to her community. Every step of her life takes her away from her family and even from the people who loved her.

\section{INDEX}

Mots-clés : autobiographie, écriture de soi, écriture de l'intime, exil intérieur, exil géographique Keywords : autobiography, self-expression, the expression of intimity, internal exile, geographically exile

\section{AUTEUR}

\section{FIZIA HAYETTE BOULAHBEL MOKHTARI.}

Doctorante Université Alger 2

Maitre Assistante classe A. Université de Béjaia 